\title{
Genomic evolution of uveal melanoma arising in ocular melanocytosis
}

\author{
Michael A. Durante, ${ }^{1,2,3,5}$ Matthew G. Field, ${ }^{1,2,3,5}$ Margaret I. Sanchez, ${ }^{1,2,3}$ \\ Kyle R. Covington, ${ }^{4}$ Christina L. Decatur, ${ }^{1,2,3}$ Sander R. Dubovy, ${ }^{1}$ \\ and J. William Harbour ${ }^{1,2,3}$ \\ ${ }^{1}$ Bascom Palmer Eye Institute, ${ }^{2}$ Sylvester Comprehensive Cancer Center, ${ }^{3}$ Interdisciplinary Stem Cell Institute, \\ University of Miami Miller School of Medicine, Miami, Florida 33136, USA; ${ }^{4}$ Castle Biosciences Inc., \\ Friendswood, Texas 77546, USA
}

\begin{abstract}
Ocular melanocytosis is the most important predisposing condition for the eye cancer uveal melanoma (UM). Here, we present a patient who developed UM arising within ocular melanocytosis who was treated with enucleation (eye removal), which provided an invaluable opportunity to interrogate both the UM and adjacent uveal tissue containing the melanocytosis using whole-exome and deep-targeted sequencing. This analysis revealed a clonal PLCB4 mutation in the melanocytosis, confirming that this is indeed a neoplastic condition and explaining why it predisposes to UM. This mutation was present in $100 \%$ of analyzed UM cells, indicating that a PLCB4-mutant cell gave rise to the UM. The earliest aberrations specific to the tumor were loss of Chromosomes 1p, 3, and 9p, which were present in virtually all tumor cells. A mutation in BAP1 arose later on the other copy of Chromosome 3 in a tumor subclone, followed by a gain of Chromosome $8 q$. These findings provide a mechanistic explanation for the well-known clinical association between ocular melanocytosis and UM by showing that this predisposing condition introduces the first "hit" and thereby increases the stochastic likelihood of acquiring further aberrations leading to UM.
\end{abstract}

Corresponding author: harbour@miami.edu

(C) 2019 Durante et al. This article is distributed under the terms of the Creative Commons Attribution-NonCommercial License, which permits reuse and redistribution, except for commercial purposes, provided that the original author and source are credited.

Ontology term: choroidal melanoma

Published by Cold Spring Harbor Laboratory Press

doi:10.1101/mcs.a004051

\section{INTRODUCTION}

Ocular melanocytosis is characterized by an excess of melanocytes in the uveal tract, comprising the choroid, ciliary body, and iris, and it has been speculated to represent a congenital nevus (Yanoff and Zimmerman 1967). This condition is usually nonhereditary and unilateral and manifests with brown-gray episcleral patches and dark pigmentation of the ocular fundus, sometimes accompanied by iris heterochromia. When associated with periocular cutaneous hyperpigmentation, it is referred to as nevus of Ota (Teekhasaenee et al. 1990). Ocular melanocytosis is the strongest predisposing factor for uveal melanoma (UM), increasing its risk from $\sim 1$ in 230,000 to $\sim 1$ in 400 (Singh et al. 1998), and its presence doubles the risk of metastasis from UM (Shields et al. 2013).

$\mathrm{UM}$ is the most common primary eye cancer and frequently results in metastatic death (Harbour and Shih 2018). UM exhibits a distinctive molecular landscape consisting of two mutational "nodes." The first is characterized by mutually exclusive gain-of-function point mutations in members of the Gq signaling pathway (GNAQ, GNA11, CYSLTR2, and PLCB4), which trigger constitutive activation of proliferative and survival signals (Van Raamsdonk et al. 2009, 2010; Johansson et al. 2016; Moore et al. 2016). The second

\footnotetext{
${ }^{5}$ These authors contributed equally to this work.
} 
mutational node consists of near-mutually exclusive mutations in the tumor suppressor BAP1, the splicing factors SF3B1, SF3A1, SRSF2, SRSF7, and RBM10, and the translational initiation factor EIF1AX (Harbour et al. 2010, 2013; Martin et al. 2013; Robertson et al. 2017; Field et al. 2018). These "BSE" mutations are prognostically significant and associated with specific chromosomal copy-number aberrations (CNAs) (Field et al. 2018). Notably, mutations in BAP1, located on Chromosome 3p21, are associated with loss of heterozygosity for Chromosome $3(\mathrm{LOH} 3)$, leading to biallelic inactivation of BAP1 and poor prognosis (Harbour et al. 2010).

By the time UMs are detected, the most recent common ancestral tumor clone usually contains a full complement of mutations and CNAs, indicating that these canonical aberrations occur early during tumor evolution (Field et al. 2018). To determine the order in which these events arise would require an analysis of early precursor lesions, but tissue samples from premalignant uveal nevi are rarely available owing to the delicate location of these lesions within the eye. Here, we describe a patient with ocular melanocytosis who developed UM and was treated by enucleation (eye removal), which provided an invaluable opportunity to interrogate both the tumor and precursor melanocytosis. Next-generation sequencing followed by bioinformatic analysis allowed us to elucidate the early genomic evolutionary events leading to UM.

\section{RESULTS}

A 56-yr-old white female presented with pain and blurred vision in her left eye, in which the best-corrected visual acuity had dropped to 20/800. External examination revealed diffuse episcleral pigmentation of the left eye (Fig. 1A), consistent with ocular melanocytosis.
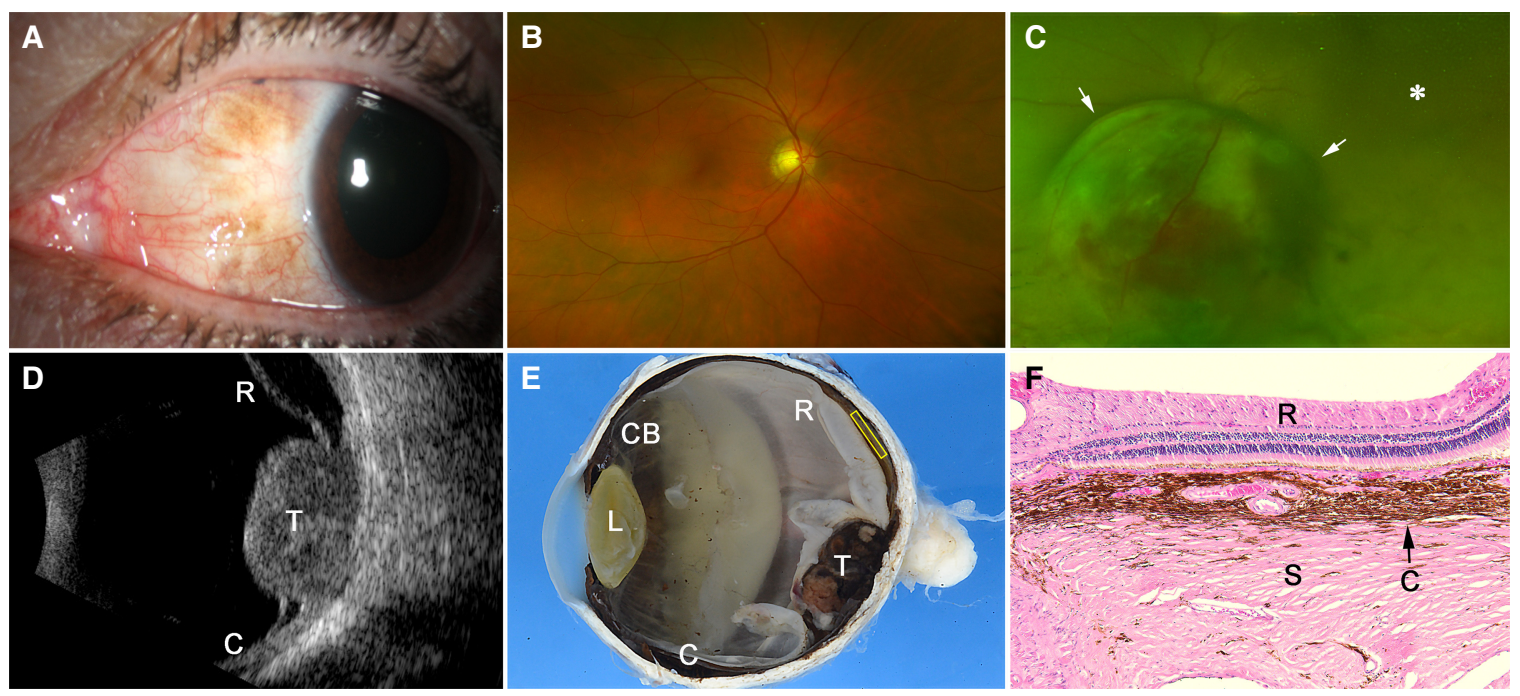

Figure 1. Clinical and pathologic findings in uveal melanoma (UM) associated with ocular melanocytosis. (A) Episcleral brown pigmented patches in the left eye, consistent with ocular melanocytosis. (B) Color fundus photograph of the normal right eye, $(C)$ in contrast to the darkly pigmented fundus (asterisk) and associated UM (arrows) in the left eye. (D) B-scan ultrasonography of the UM with associated exudative retinal detachment. $(E)$ Enucleated left eye, with dark pigmentation and thickening of the choroid and ciliary body. The region of ocular melanocytosis within the choroid that was sampled for genomic analysis is indicated by the yellow box. (F) Histopathologic examination reveals the choroid filled with heavily pigmented melanocytes (arrow) is typical for ocular melanocytosis (hematoxylin \& eosin, original magnification 20x). (C) Choroid, (CB) ciliary body, $(\mathrm{L})$ lens, $(\mathrm{R})$ retina, $(\mathrm{S})$ sclera, $(\mathrm{T})$ tumor. 
Fundus ophthalmoscopic examination showed normal fundus pigmentation in the right eye (Fig. 1B), in contrast to diffuse fundus hyperpigmentation in the left eye accompanied by a darkly pigmented choroidal tumor (Fig. 1C). Ultrasonographic examination of the left eye revealed a pedunculated choroidal tumor measuring $14.6 \times 14.4 \times 7.9 \mathrm{~mm}$, with low internal reflectivity, spontaneous vascular pulsations, and a surrounding exudative retinal detachment, which are typical for UM (Fig. 1D). Following enucleation, the eye was immediately dissected in the operating room to obtain and snap-freeze samples from the tumor and nontumorous melanocytosis. Histopathological evaluation revealed a marked overabundance of heavily pigmented melanocytes throughout the uveal tract, along with the UM arising from the choroid (Fig. 1E,F).

Molecular prognostic testing of the tumor revealed a class 2 GEP, associated with high metastatic risk (Onken et al. 2012). Whole-exome sequencing (WES) followed by mutational analysis revealed a deleterious somatic single-nucleotide alteration (p.D630Y) in PLCB4 in both the tumor and melanocytosis but not in the blood (Fig. 2A). An in-frame deletion (p.C320_D311del) that extended into the intronic region was detected in BAP1 in the tumor but not in the melanocytosis or blood (Table 1; Fig. 2A). Analysis of CNAs using WES data revealed loss of Chromosomes 1p, 3, and 9p and gain of Chromosome 8 (Fig. 2C), all of which were present in the tumor but not the melanocytosis.

The PLCB4 mutation was present in slightly less than one-quarter of cells comprising the melanocytosis and in $\sim 100 \%$ of analyzed tumor cells (Fig. 2B), indicating that it arose within the melanocytosis and that a PLCB4-mutant cell gave rise to the UM. LOH3 was absent from the melanocytosis but present in $\sim 100 \%$ of analyzed tumor cells, indicating that it arose early during clonal tumor expansion. By comparison, the BAP1 mutation was present in about three-quarters and the $8 \mathrm{q}$ gain in about one-quarter of the tumor cells. Although limitations in methodology did not allow detailed conclusions regarding genomic evolution, these findings imply that the BAP1 mutation and 8q gain occurred after $\mathrm{LOH} 3$, which is consistent with previous reports (Robertson et al. 2017; Field et al. 2018).

\section{DISCUSSION}

Gq pathway mutations have been reported in UM, cutaneous blue nevi, and central nervous system melanocytic neoplasms (Küsters-Vandevelde et al. 2010; Van Raamsdonk et al. 2010; Vivancos et al. 2016). However, this is the first direct demonstration of a Gq pathway mutation in the nonmalignant uveal tissue giving rise to melanoma in an eye with ocular melanocytosis. Further, it is the first report of a PLCB4 mutation in any form of ocular or oculodermal melanocytosis. As such, this case is quite distinct from a recent report of cutaneous melanoma arising within nevus of Ota (Vivancos et al. 2016), which has different demographics and clinical features than ocular melanocytosis. Recently, we identified a second case of ocular melanocytosis in which the nonmalignant uveal tissue harbored a somatic GNA11 mutation (data not shown), suggesting that Gq pathway mutations may be characteristic of this condition. This oncogenic "first hit" mutation could explain the increased risk of UM associated with ocular melanocytosis and confirms that this condition represents a congenital nevus rather than simply an excess of normal melanocytes. The absence of a germline mutation is consistent with the lack of hereditary transmission in most patients with this condition.

We recently analyzed 151 primary UMs and showed that all of the canonical mutations and CNAs usually arose early in tumor evolution, making it difficult to discriminate the relative order in which these aberrations accrued (Field et al. 2018). This case allowed a more detailed exploration into the evolutionary dynamics of UM (Fig. 3). The PLCB4 mutation in the precursor melanocytosis was clearly the initiating event but could not effectuate malignant transformation without additional aberrations. This finding is particularly interesting 
A

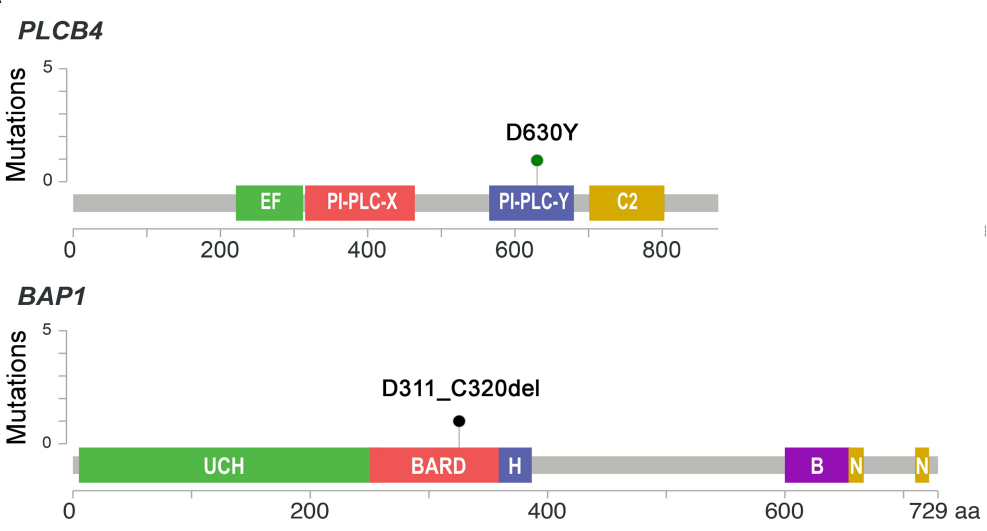

B

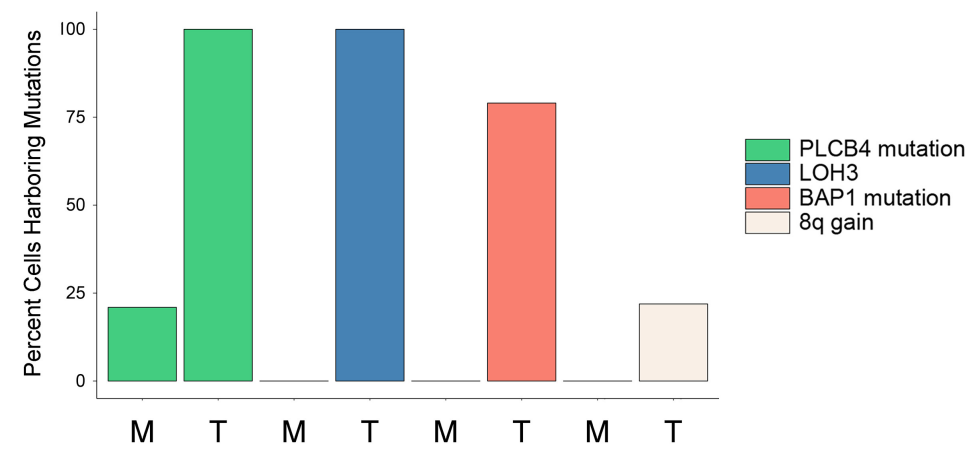

C
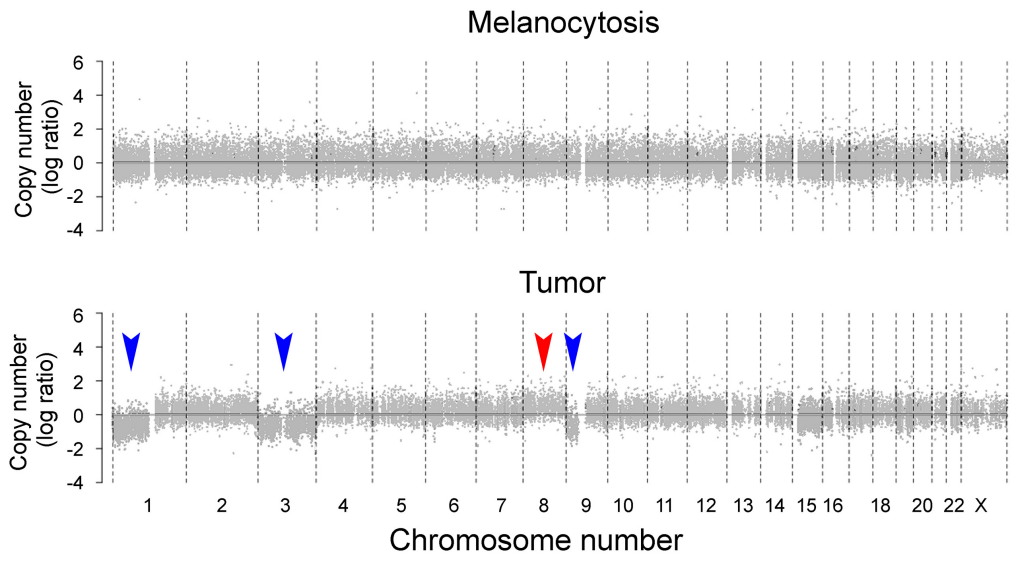

Figure 2. Genomic evolutionary analysis of ocular melanocytosis and associated uveal melanoma. (A) Lollipop plots that depict the amino acid changes resulting from the PLCB4 (p.D630Y) and BAP1 (p.C320_D311del) mutations in the tumor. (EF) Phosphoinositide-specific phospholipase $\mathrm{C}$ domain, (PI-PLC-X) phosphatidylinositol-specific phospholipase $\mathrm{C}$, X domain, (PI-PLC-Y) phosphatidylinositol-specific phospholipase $\mathrm{C}-\mathrm{Y}$ domain, (C2) $\mathrm{C} 2$ domain, $(\mathrm{CH})$ ubiquitin carboxy-terminal hydrolase catalytic domain, (BARD) BRCA1-associated RING domain, (H) HCF1-binding motif, (B) BRCA1-binding domain, (N) nuclear localization sequences. (B) Percentage of cells carrying a driver mutation or chromosomal aberration in the ocular melanocytosis versus the tumor. (M) Melanocytosis, (T) tumor. (C) Copy-number plots for melanocytosis and tumor samples, respectively, as determined by THetA2. Whereas the melanocytosis showed no copy-number alterations, the tumor showed loss of Chromosomes 1p, 3, and 9p (blue arrowheads) and a smaller subclone with gain of Chromosome 8 (red arrowhead). 


\begin{tabular}{|c|c|c|c|c|c|c|c|}
\hline Gene & Genomic location & HGVS cDNA & HGVS protein & Variant type & Variant interpretation & COSMIC ID & Zygosity \\
\hline PLCB4 & $\begin{array}{c}\text { Chromosome 20, } \\
\text { NC_000020.10 }\end{array}$ & c. $1888 \mathrm{G}>\mathrm{T}$ & p.D630Y & Substitution & Pathogenic & COSM1666823 & Heterozygous \\
\hline BAP1 & $\begin{array}{l}\text { Chromosome 3, } \\
\text { NC_000003.11 }\end{array}$ & $\begin{array}{l}\text { c.932-8_960 } \\
\text { delTGTCTCAGATGGTGCAGA } \\
\text { GGAGGCGGCTGGTTCATGC }\end{array}$ & p.D311_C320del & Deletion & Pathogenic & None & Hemizygous \\
\hline
\end{tabular}

because mutant GNAQ signals to PKC through PLC $\beta$ and thereby activates the MAPK pathway (Chen et al. 2017). The earliest events specific to the tumor were loss of Chromosomes $1 p, 3$, and 9p, which were present in virtually all tumor cells. Additionally, by producing haploinsufficiency for BAP1, LOH3 may have created a selective pressure to inactivate the other BAP1 allele, a requisite event for conversion to the highly metastatic class 2 GEP (Harbour et al. 2010; Onken et al. 2012). Thus, LOH3 could represent a "threshold" event in malignant transformation. Gain of $8 q$ was a late event and, hence, apparently not required for malignant transformation but likely providing a selective advantage during tumor progression.

These insights may help to address persistent challenges in the field. For example, the failure of targeted therapies aimed at inhibiting the Gq pathway (Carvajal et al. 2018) might be explained by the inability of these mutations to drive malignant tumor growth without additional genomic aberrations. Further, some UMs with good prognosis exhibit $\mathrm{LOH} 3$ yet retain a wild-type BAP1 allele and class 1 GEP (Onken et al. 2012; Field et al. 2018), suggesting that biallelic loss of BAP1 is required for conversion to the class 2 GEP and that $\mathrm{LOH} 3$ alone is insufficient to confer high metastatic risk.

\section{METHODS}

\section{Sample Collection}

Tissue samples were subjected to DNA extraction using the Wizard Genomic DNA Purification kit (Promega) and RNA extraction using the PicoPure RNA Isolation kit (Thermo

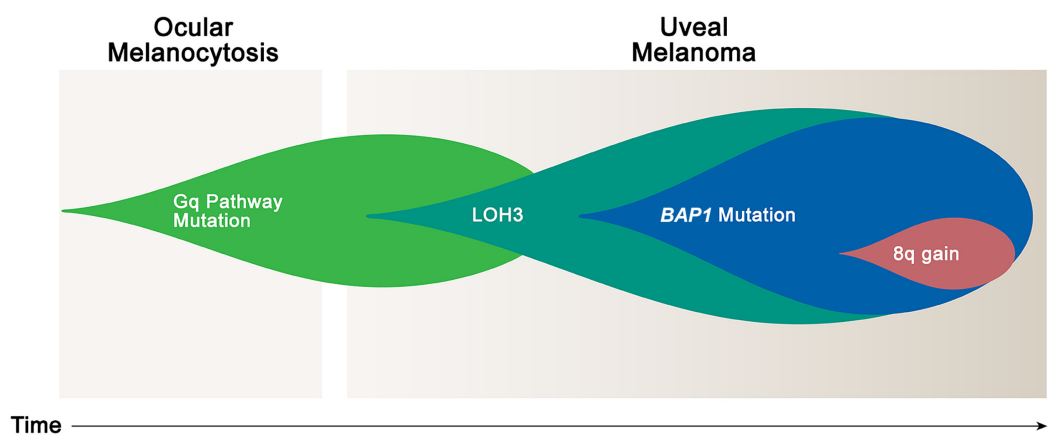

Figure 3. Qualitative tumor evolution diagram of ocular melanocytosis giving risk to UM. The proposed genomic evolution from melanocytosis to UM, in this case, is presented. The Gq pathway mutation (PLCB4) was the earliest event, arising in the ocular melanocytosis and present in all tumor cells. Loss of heterozygosity for Chromosome 3 (LOH3) was absent from the melanocytosis but present in all tumor cells, and it was followed by mutation of BAP1 on the other copy of Chromosome 3. These events resulting in biallelic BAP1 deficiency occurred close in evolutionary time and may represent a threshold event in malignant transformation by converting the tumor to the high metastatic risk class 2 gene expression profile (GEP). A small subclone of Chromosome 8 gain is also observed and depicted as arising from the BAP1 subclone, although it could also have arisen in a separate subclone. For clarity, the loss of Chromosomes $1 p$ and $9 p$ is not included in the diagram. 


\begin{tabular}{llll}
\hline Table 2. Whole-exome sequencing metrics & & & \\
\hline & \multicolumn{1}{c}{ Blood } & Melanocytosis & Tumor \\
\hline Mean depth of coverage & $78 \times$ & $56 \times$ & $56 \times$ \\
Percentage of exome with at least 1 x coverage & $100 \%$ & $99 \%$ & $99 \%$ \\
Depth of coverage at reported PLCB4 variant & N/A & $83 \times$ & $178 \times$ \\
Depth of coverage at reported BAP1 variant & N/A & N/A & $30 \times$ \\
Total reads & $100,666,026$ & $80,33,8148$ & $164,244,502$ \\
Total mapped reads & $97,070,265$ & $77,365,636$ & $156,225,641$ \\
\hline
\end{tabular}

(N/A) Not applicable.

Fisher Scientific). DNA from peripheral blood leukocytes was extracted using the QuickGene DNA whole blood kit S (Fujifilm).

\section{Whole-Exome Sequencing and Validation}

WES was performed as previously described (Field et al. 2018), using the Agilent SureSelect XT Human All Exon V5 kit with 100-bp paired-end sequencing on the Illumina HiSeq 2500 sequencer (Table 2). WES mutation results were validated using the DecisionDx-UMseq targeted next-generation sequencing panel (Castle Biosciences, Inc.). Molecular prognostic classification of the tumor was performed by GEP as part of routine clinical management using the DecisionDx-UM test (Castle Biosciences, Inc.). The GEP test uses a 15-gene PCR assay comprising of 12 discriminating genes, two control genes, and expression of PRAME, a gene that provides independent prognostic information. This test can be performed on fineneedle aspirate biopsy samples and stratifies UM patients in the low-, intermediate-, and high-risk groups (Onken et al. 2010).

\section{Bioinformatic Analysis}

FASTQ files containing WES data were subjected to quality control using FASTOC (v0.11.3), trimmed and aligned to the human genome build hg19/GRCH37 using Novoalign (v3.04.06), marked for duplicates using Picard (v1.128), realigned around small and large indels using ABRA (v0.94c) (Mose et al. 2014), and then read mate fixed and analyzed for coverage statistics using Picard. Variant calling for SNPs and indels was performed using MuTect2 (GATK 2016-01-25 nightly build) (Cibulskis et al. 2013) comparing the tumor and melanocytosis regions to matched blood. Lollipop plots were generated using MutationMapper (http://www .cbioportal.org/mutation_mapper.jsp), and domain information was annotated from a previous publication (Field et al. 2018). CNAs were assessed from WES data using CNVKit (v0.7.5, v0.7.10.dev0) (Talevich et al. 2016) and b-allele frequency plots were assessed from WES using THetA2 (v0.7) (Oesper et al. 2014). Clonality of CNAs was assessed using cgpBattenberg, as previously described (Nik-Zainal et al. 2012; Field et al. 2018), and depicted using a qualitative tumor clone evolution diagram (Krzywinski 2016).

\section{ADDITIONAL INFORMATION}

\section{Data Deposition and Access}

All sequencing data generated have been deposited in dbGaP (https://www.ncbi.nlm.nih .gov/gap) under accession code phs001835.v1.p1. The reported variants were submitted to ClinVar (https://ncbi.nlm.nih.gov/clinvar/) and can be found under accession numbers SCV000920585 and SCV000920586. 
COLD SPRING HARBOR Molecular Case Studies
Genomic evolution of uveal melanoma
Competing Interest Statement

Dr. Harbour is the inventor of intellectual property discussed in this study. Dr. Harbour is a paid consultant for Castle

Biosciences, licensee of this intellectual property, and he receives royalties from its commercialization. Dr.

Covington is an employee of Castle Biosciences. No other authors declare a potential competing interest.

Received February 5, 2019; accepted in revised form May 17, 2019.

\section{Ethics Statement}

This study was approved by the Institutional Review Board of the University of Miami School of Medicine. Patient informed consent was obtained.

\section{Acknowledgments}

We are grateful to the patient who generously contributed samples for this research. We acknowledge the support of the Biostatistics \& Bioinformatics and Oncogenomics Shared Resources at the Sylvester Comprehensive Cancer Center, the John P. Hussman Institute for Human Genomics, and the Center for Computational Science High Performance Computing Group at the University of Miami.

\section{Author Contributions}

M.A.D. and M.G.F. analyzed and interpreted the data and wrote the manuscript. M.I.S. collected and prepared samples. K.R.C. analyzed and interpreted the data. C.L.D. maintained the clinical database and oversaw regulatory compliance. S.R.D. performed the histopathologic analysis and interpreted findings. J.W.H. designed and led the project, provided clinical samples, interpreted the data, and wrote the manuscript. All authors have read and approved the final manuscript.

\section{Funding}

This work was supported by National Cancer Institute grants R01 CA125970 (J.W.H.) and F30 CA206430 (M.G.F.), Research to Prevent Blindness, Inc. Senior Scientific Investigator Award (J.W.H.), the University of Miami Sheila and David Fuente Graduate Program in Cancer Biology (M.A.D., M.G.F.), the Center for Computational Science Fellowship (M.A.D., M.G.F.), and a generous gift from Dr. Mark J. Daily (J.W.H). The Bascom Palmer Eye Institute also received funding from National Institutes of Health Core Grant P30EY014801 and a Research to Prevent Blindness Unrestricted Grant.

\section{REFERENCES}

Carvajal RD, Piperno-Neumann S, Kapiteijn E, Chapman PB, Frank S, Joshua AM, Piulats JM, Wolter P, Cocquyt V, Chmielowski B, et al. 2018. Selumetinib in combination with dacarbazine in patients with metastatic uveal melanoma: a phase III, multicenter, randomized trial (SUMIT). J Clin Oncol 36: 1232-1239. doi:10.1200/JCO.2017.74.1090

Chen X, Wu Q, Depeille P, Chen P, Thornton S, Kalirai H, Coupland SE, Roose JP, Bastian BC. 2017. RasGRP3 mediates MAPK pathway activation in GNAQ mutant uveal melanoma. Cancer Cell 31: 685-696.e6. doi:10 .1016/j.ccell.2017.04.002

Cibulskis K, Lawrence MS, Carter SL, Sivachenko A, Jaffe D, Sougnez C, Gabriel S, Meyerson M, Lander ES, Getz G. 2013. Sensitive detection of somatic point mutations in impure and heterogeneous cancer samples. Nat Biotechnol 31: 213-219. doi:10.1038/nbt.2514

Field MG, Durante MA, Anbunathan H, Cai LZ, Decatur CL, Bowcock AM, Kurtenbach S, Harbour JW. 2018. Punctuated evolution of canonical genomic aberrations in uveal melanoma. Nat Commun 9: 116. doi:10 .1038/s41467-017-02428-w

Harbour JW, Shih HA. 2018. Initial management of uveal and conjunctival melanomas. In UpToDate (ed. Atkins MB, Berman RS). Waltham, MA (Accessed on March 5, 2019).

Harbour JW, Onken MD, Roberson ED, Duan S, Cao L, Worley LA, Council ML, Matatall KA, Helms C, Bowcock AM. 2010. Frequent mutation of BAP1 in metastasizing uveal melanomas. Science 330: 1410-1413. doi:10 .1126/science.1194472

Harbour JW, Roberson ED, Anbunathan H, Onken MD, Worley LA, Bowcock AM. 2013. Recurrent mutations at codon 625 of the splicing factor SF3B1 in uveal melanoma. Nat Genet 45: 133-135. doi:10.1038/ng.2523

Johansson P, Aoude LG, Wadt K, Glasson WJ, Warrier SK, Hewitt AW, Kiilgaard JF, Heegaard S, Isaacs T, Franchina $M$, et al. 2016. Deep sequencing of uveal melanoma identifies a recurrent mutation in PLCB4. Oncotarget 7: 4624-4631. doi:10.18632/oncotarget.6614 
Krzywinski M. 2016. Visualizing clonal evolution in cancer. Mol Cell 62: 652-656. doi:10.1016/j.molcel.2016 05.025

Küsters-Vandevelde HV, Klaasen A, Küsters B, Groenen PJ, van Engen-van Grunsven IA, van Dijk MR, Reifenberger G, Wesseling P, Blokx WA. 2010. Activating mutations of the GNAQ gene: a frequent event in primary melanocytic neoplasms of the central nervous system. Acta Neuropathol 119: 317-323. doi:10 1007/s00401-009-0611-3

Martin M, Maßhöfer L, Temming P, Rahmann S, Metz C, Bornfeld N, van de Nes J, Klein-Hitpass L, Hinnebusch $A G$, Horsthemke B, et al. 2013. Exome sequencing identifies recurrent somatic mutations in EIF1AX and SF3B1 in uveal melanoma with disomy 3. Nat Genet 45: 933-936. doi:10.1038/ng.2674

Moore AR, Ceraudo E, Sher JJ, Guan Y, Shoushtari AN, Chang MT, Zhang JQ, Walczak EG, Kazmi MA, Taylor BS, et al. 2016. Recurrent activating mutations of G-protein-coupled receptor CYSLTR2 in uveal melanoma. Nat Genet 48: 675-680. doi:10.1038/ng.3549

Mose LE, Wilkerson MD, Hayes DN, Perou CM, Parker JS. 2014. ABRA: improved coding indel detection via assembly-based realignment. Bioinformatics 30: 2813-2815. doi:10.1093/bioinformatics/btu376

Nik-Zainal S, Van Loo P, Wedge DC, Alexandrov LB, Greenman CD, Lau KW, Raine K, Jones D, Marshall J, Ramakrishna M, et al. 2012. The life history of 21 breast cancers. Cell 149: 994-1007. doi:10.1016/j.cell 2012.04.023

Oesper L, Satas G, Raphael BJ. 2014. Quantifying tumor heterogeneity in whole-genome and whole-exome sequencing data. Bioinformatics 30: 3532-3540. doi:10.1093/bioinformatics/btu651

Onken MD, Worley LA, Tuscan MD, Harbour JW. 2010. An accurate, clinically feasible multi-gene expression assay for predicting metastasis in uveal melanoma. J Mol Diagn 12: 461-468. doi:10.2353/jmoldx.2010 .090220

Onken MD, Worley LA, Char DH, Augsburger JJ, Correa ZM, Nudleman E, Aaberg TM, Altaweel MM, Bardenstein DS, Finger PT, et al. 2012. Collaborative Ocular Oncology Group report number 1: prospective validation of a multi-gene prognostic assay in uveal melanoma. Ophthalmology 119: 1596-1603. doi:10.1016/j.ophtha.2012.02.017

Robertson AG, Shih J, Yau C, Gibb EA, Oba J, Mungall KL, Hess JM, Uzunangelov V, Walter V, Danilova L, et al. 2017. Integrative analysis identifies four molecular and clinical subsets in uveal melanoma. Cancer Cell 32: 204-220.e15. doi:10.1016/j.ccell.2017.07.003

Shields CL, Kaliki S, Livesey M, Walker B, Garoon R, Bucci M, Feinstein E, Pesch A, Gonzalez C, Lally SE, et al. 2013. Association of ocular and oculodermal melanocytosis with the rate of uveal melanoma metastasis: analysis of 7872 consecutive eyes. JAMA Ophthalmol 131: 993-1003. doi:10.1001/jamaophthalmol .2013 .129

Singh AD, De Potter P, Fijal BA, Shields CL, Shields JA, Elston RC. 1998. Lifetime prevalence of uveal melanoma in white patients with oculo(dermal) melanocytosis. Ophthalmology 105: 195-198. doi:10.1016/ S0161-6420(98)92205-9

Talevich E, Shain AH, Botton T, Bastian BC. 2016. CNVkit: genome-wide copy number detection and visualization from targeted DNA sequencing. PLoS Comput Biol 12: e1004873. doi:10.1371/journal.pcbi 1004873

Teekhasaenee C, Ritch R, Rutnin U, Leelawongs N. 1990. Ocular findings in oculodermal melanocytosis. Arch Ophthalmol 108: 1114-1120. doi:10.1001/archopht.1990.01070100070037

Van Raamsdonk CD, Bezrookove V, Green G, Bauer J, Gaugler L, O'Brien JM, Simpson EM, Barsh GS, Bastian BC. 2009. Frequent somatic mutations of GNAQ in uveal melanoma and blue nevi. Nature 457: 599-602. doi:10.1038/nature07586

Van Raamsdonk CD, Griewank KG, Crosby MB, Garrido MC, Vemula S, Wiesner T, Obenauf AC, Wackernagel W, Green G, Bouvier N, et al. 2010. Mutations in GNA11 in uveal melanoma. N Engl J Med 363: 21912199. doi:10.1056/NEJMoa1000584

Vivancos A, Caratú G, Matito J, Muñoz E, Ferrer B, Hernández-Losa J, Bodet D, Pérez-Alea M, Cortés J, GarciaPatos V, et al. 2016. Genetic evolution of nevus of Ota reveals clonal heterogeneity acquiring BAP1 and TP53 mutations. Pigment Cell Melanoma Res 29: 247-253. doi:10.1111/pcmr.12452

Yanoff M, Zimmerman LE. 1967. Histogenesis of malignant melanomas of the uvea. III. The relationship of congenital ocular melanocytosis and neurofibromatosis in uveal melanomas. Arch Ophthalmol 77: 331-336. doi:10.1001/archopht.1967.00980020333007 


\section{COLD SPRING HARBOR Molecular Case Studies}

\section{Genomic evolution of uveal melanoma arising in ocular melanocytosis}

Michael A. Durante, Matthew G. Field, Margaret I. Sanchez, et al.

Cold Spring Harb Mol Case Stud 2019, 5: a004051 originally published online June 11, 2019 Access the most recent version at doi:10.1101/mcs.a004051

References This article cites 25 articles, 1 of which can be accessed free at: http://molecularcasestudies.cshlp.org/content/5/4/a004051.full.html\#ref-list-1

License This article is distributed under the terms of the Creative Commons Attribution-NonCommercial License, which permits reuse and redistribution, except for commercial purposes, provided that the original author and source are credited.

Email Alerting Receive free email alerts when new articles cite this article - sign up in the box at the Service top right corner of the article or click here. 